\title{
ON THE MEAN EXIT TIME FROM A MINIMAL SUBMANIFOLD
}

\author{
STEEN MARKVORSEN
}

\begin{abstract}
Let $M^{m}$ be an immersed minimal submanifold of a Riemannian manifold $N^{n}$, and consider the Brownian motion on a regular ball $\Omega_{R} \subset M$ with exterior radius $R$. The mean exit time for the motion from a point $x \in \Omega_{R}$ is called $E_{R}(x)$. In this paper we find sharp support functions for $E_{R}(x)$ in the following distinct cases. The support is from below if the sectional curvatures of $N$ are bounded from below by a nonnegative constant and the support is from above if the sectional curvatures of $N$ are bounded from above by a nonpositive constant. It follows that the minimal submanifolds of $\mathbf{R}^{n}$ all have the same mean exit time function and we show that this function actually characterizes the minimal hypersurfaces in the set of all hypersurfaces of $\mathbf{R}^{n}$.
\end{abstract}

\section{Introduction}

Let $M^{m}$ be an immersed (not necessarily minimal) submanifold of a complete Riemannian manifold $N^{n}$. The distance function on $N$ is denoted by $r$ so that $\operatorname{dist}_{N}(p, x)=r_{p}(x)$ for all $p, x$ in $N$. We now fix $p \in M \subset N$ and define a regular domain $\Omega_{R}(p) \subset M$ to be a smooth connected component of $B_{R}(p) \cap M^{m}$ which contains $p$. Here $B_{R}(p)$ is the geodesic $R$-ball around $p$ in $N$ subject to the usual restriction that $R<\min \left\{i_{N}(p), \pi \sqrt{\kappa}\right\}$, where $\kappa$ is the supremum of the sectional curvatures of $N$, and $i_{N}(p)$ is the injectivity radius of $N$ from $p$.

We now consider the Brownian motion on the domain $\Omega_{R}$ (cf. [4]) and denote by $E_{R}(x)$ the mean time of first exit from $\Omega_{R}$ for a Brownian particle starting at $x \in \Omega_{R}$. We want to compare $E_{R}(x)$ with the mean exit time function $\tilde{E}_{R}^{b}(\tilde{x})$ on the space form $R$-ball $\tilde{B}_{R}^{m}(\tilde{p})$ of dimension $m=\operatorname{dim} M$ and constant curvature $b \in \mathbf{R}$. Since $\tilde{B}_{R}$ has maximal isotropy at the center $\tilde{p}$, we have that $\tilde{E}_{R}$ only depends on the distance of $\tilde{x}$ from $\tilde{p}$. Hence we may, and do, write $\tilde{E}_{R}^{b}(\tilde{x})=\tilde{\mathscr{E}}_{R}^{b}\left(r_{\tilde{p}}(\tilde{x})\right)=\tilde{\mathscr{E}}_{R}^{b}(r)$. In order to compare $E$ and $\tilde{E}$ we transplant $\tilde{E}$ to $\Omega_{R}$ by the following definition:

$$
\tilde{E}_{R}^{b}: \Omega_{R} \rightarrow \mathbf{R} ; \quad \tilde{E}_{R}^{b}(x)=\left(\tilde{\mathscr{E}}_{R}^{b} \circ r_{p}\right)(x) .
$$

Our main result can now be formulated as follows. 
Theorem 1. Let $M^{m}$ be a minimally immersed submanifold of $N^{n}$ and let $\Omega_{R}(p)$ be a regular domain on $M$.

(i) If the sectional curvatures $K_{N}$ of $N$ satisfy $K_{N} \geq b \geq 0$, then $E_{R}(x) \geq$ $\tilde{E}_{R}^{b}(x)$ for all $x \in \Omega_{R}$.

(ii) If $K_{N} \leq b \leq 0$, then $E_{R}(x) \leq \tilde{E}_{R}^{b}(x)$ for all $x \in \Omega_{R}$.

Remark. This type of theorem was first proved by Debiard, Gaveau and Mazet in [3] where they used techniques different from ours to obtain bounds on the mean exit times from codimension- 0 domains of $N$. The setting in Theorem 1 is more general in the sense that we have no a priori control on the sectional curvatures of the domains $\Omega_{R}$ in question.

A fundamental observation of Dynkin (cf. [4, vol. 2, p. 51]) states that the function $E_{R}(x)$ satisfies the Poisson equation on $\Omega_{R}$ with Dirichlet boundary data, i.e.,

$$
\Delta_{\Omega} E_{R}=-1,\left.\quad E_{R}\right|_{\partial \Omega_{R}} \equiv 0,
$$

where $\Delta_{\Omega}$ is the induced Laplacian on $\Omega_{R}$ defined by $\Delta_{\Omega}=\operatorname{div}_{\Omega} \circ \operatorname{grad}_{\Omega}=$ $\operatorname{trace}_{\Omega} \circ \operatorname{Hess}_{\Omega}$.

If we let $G(x, y)$ denote the Green's functions with Dirichlet boundary conditions on $\Omega_{R}$, then every smooth function $f$ on $\Omega_{R}$ satisfies

$$
f(x)=-\int_{\Omega_{R}} G(x, y) \Delta f(y) d y-\int_{\partial \Omega_{R}} \frac{\partial G}{\partial \xi}(x, y) f(y) d y .
$$

In particular, if we set $f=E_{R}$ we get by (1.1)

$$
E_{R}(x)=\int_{\Omega_{R}} G(x, y) d y .
$$

On the other hand, the Green's functions are given by

$$
G(x, y)=\int_{0}^{\infty} \mathscr{H}(x, y, t) d t
$$

where $\mathscr{H}$ is the Dirichlet heat kernel on $\Omega_{R}$. Thus

$$
E_{R}(x)=\int_{\Omega_{R}} \int_{0}^{\infty} \mathscr{H}(x, y, t) d t d y
$$

and similarly

$$
\tilde{E}_{R}^{b}(\tilde{x})=\int_{\tilde{B}_{R}} \int_{0}^{\infty} \tilde{\mathscr{H}}_{R}^{b}(\tilde{x}, \tilde{y}, t) d t d \tilde{y} .
$$

In [8] we showed that under the assumptions of Theorem 1(ii) we have

$$
\mathscr{H}(p, y, t) \leq \tilde{\mathscr{H}}_{R}^{b}\left(r_{p}(y), t\right),
$$

and also

$$
\mathscr{K}(p, y, t) \leq \tilde{\mathscr{K}}_{R}^{b}\left(r_{p}(y), t\right)
$$


where $\mathscr{K}$ and $\tilde{\mathscr{K}}$ are the Neumann heat kernels on $\Omega_{R}(p)$ and $\tilde{B}_{R}(\tilde{p})$ respectively. From these latter kernels one may extract the volumes of the respective domains (cf. [2]) and obtain

$$
\int_{\Omega_{R}} d y \geq \int_{\tilde{B}_{R}} d \tilde{y}
$$

It is now natural to ask for situations where inequalities of type (1.3) and (1.5) balance each other so as to give

$$
E_{R}(x)=\tilde{E}_{R}^{b}(x) \quad \text { for all } x \in \Omega_{R} .
$$

With this in mind we show the following gap phenomenon for minimal submanifolds of constant curvature space forms: The condition $(*)$ is a Bernstein type condition in every nonflat space form in the sense that $\Omega_{R}$ (and in fact all of $M^{m}$ ) must be totally geodesic if it is minimal and satisfies $(*)$. In contrast, (*) is always satisfied in $\mathbf{R}^{n}$, where it even characterizes minimal hypersurfaces in the set of all hypersurfaces. To be precise we have the following theorems.

Theorem 2. Under the assumptions of Theorem 1, suppose further that (*) is fulfilled and that $b \neq 0$. Then $\Omega_{R}$ is a minimal cone in $N^{n}$ and hence, if $N^{n}$ is actually a space form of constant curvature $b$, then $M^{m}$ is a totally geodesic submanifold of $N^{n}$.

Theorem 3. Let $\Omega_{R}$ be a regular domain of a minimal submanifold of $\mathbf{R}^{n}$. Then $(*)$ is satisfied. Conversely, if $(*)$ is satisfied for all $x$ in a regular domain $\Omega_{R}$ of a hypersurface in $\mathbf{R}^{n}$, then $\Omega_{R}$ is minimal.

We note here that Theorem 3 may be viewed as a semiglobal generalization of the following local result which is due to L. Karp and M. Pinsky (cf. [7]).

Theorem A. Let $M^{n-1}$ be a hypersurface of $\mathbf{R}^{n}$, and consider the domains $\Omega_{\epsilon}(p) \subset M$ for some fixed $p \in M$. If $E_{\epsilon}(p)=\tilde{E}_{\epsilon}^{0}(p)$ for all sufficiently small $\epsilon>0$, then the mean curvature of $M$ at $p$ is zero.

Remark. In order to construct the functional dependence of $E_{\epsilon}(p)$ on $\epsilon$ in this theorem it is in principle necessary to solve an infinite number of Poisson equations (one for every $\epsilon$ ), whereas it is only necessary to solve a single Poisson equation (i.e., (1.1)) to check condition (*) for Theorem 3.

\section{Some preliminary inequalities}

The following Hessian comparison theorem is well known and may be obtained from standard index comparison theory (cf. [1]).

Lemma 1. If $K_{N} \leq b$ (respectively $K_{N} \geq b$ ), $b \in \mathbf{R}$, then for every unit vector $X$ in the tangent bundle of $B_{R}(p)-p$ we have

$$
\operatorname{Hess}_{N}\left(r_{p}\right)(X, X) \geq(\leq) h_{b}\left(r_{p}\right)\left(1-\left\langle\operatorname{grad}_{N} r_{p}, X\right\rangle^{2}\right) \text {, }
$$


where $h_{b}(r)$ is the constant mean curvature of any distance sphere of radius $r$ in a space form of constant curvature $b$.

Now let $F$ be a smooth function on $\mathbf{R}$. Then $F \circ r_{p}$ is a smooth function on $B_{R}(p)-p$ with a smooth restriction to $\Omega_{R}(p)-p$. A calculation along the lines of $[6, p .713]$ now gives

$$
\begin{aligned}
\operatorname{Hess}_{\Omega}(F \circ r)(X, X)= & F^{\prime \prime}(r)\left\langle\operatorname{grad}_{N}(r), X\right\rangle^{2} \\
& +F^{\prime}(r)\left(\operatorname{Hess}_{N}(r)(X, X)+\left\langle\operatorname{grad}_{N}(r), \alpha(X, X)\right\rangle\right)
\end{aligned}
$$

for all $X \in T\left(\Omega_{R}(p)-p\right)$, where $\alpha(\cdot, \cdot)$ is the second fundamental form of $\Omega$ in $N$.

Combining (2.1) and (2.2) we get

Lemma 2. Let $q \in \Omega_{R}(p)-p$ and let $\left\{X_{i}\right\}, 1 \leq i \leq m$, be an orthonormal basis of $T_{q} \Omega \subset T_{q} N$. Suppose that $F^{\prime}(r) \geq 0$ for all $r \in[0, R]$ and that $K_{N} \leq b$ (respectively $\left.K_{N} \geq b\right), b \in \mathbf{R}$. Then

$$
\begin{aligned}
\left.\Delta_{\Omega}(F \circ r)\right|_{q} \geq(\leq) & \left(F^{\prime \prime}(r)-F^{\prime}(r) h_{b}(r)\right) \cdot \sum_{j=1}^{m}\left\langle\operatorname{grad}_{N}(r), X_{j}\right\rangle^{2} \\
& +m F^{\prime}(r)\left(h_{b}(r)+\left\langle\operatorname{grad}_{N}(r), H(q)\right\rangle\right),
\end{aligned}
$$

where $H(q)$ is the mean curvature vector of $\Omega$ at $q$ in $N$.

In what follows we shall only consider functions $F=F_{b}$ defined by

$$
F_{b}(r)= \begin{cases}\frac{1}{b}(1-\cos (\sqrt{b} r)) & \text { if } b>0 \\ r^{2} / 2 & \text { if } b=0 \\ \frac{1}{b}(1-\cosh (\sqrt{-b} r)) & \text { if } b<0\end{cases}
$$

Then $F_{b} \circ r$ is a smooth function on all of $\Omega_{R}(p)$, and moreover $F_{b}^{\prime \prime}(r)=$ $F_{b}^{\prime}(r) h_{b}(r)$ so that Lemma 2 specializes to

Corollary 3. If $K_{N} \leq b$ (resp. $\left.K_{N} \geq b\right)$, then

$$
\left.\Delta_{\Omega}\left(F_{b} \circ r\right)\right|_{q} \geq(\leq) m F_{b}^{\prime}(r)\left(h_{b}(r)+p H_{\Omega}(q)\right)
$$

where we have defined $p H_{\Omega}(q)=\left\langle\operatorname{grad}_{N}(r), H(q)\right\rangle, p H_{\Omega}(p)=0$.

\section{Some properties of $\tilde{E}$}

The mean time of first exit from a space form ball of constant curvature $b$, radius $R$ and dimension $m$ is given explicitly by (cf. [3])

$$
\begin{aligned}
\tilde{\mathscr{E}}_{R}^{b}(r) & =\int_{r}^{R}\left(\theta_{b}(u) u^{m-1}\right)^{-1}\left(\int_{0}^{u} \theta_{b}(t) t^{m-1} d t\right) d u \\
& =\tilde{\mathscr{E}}_{R}^{b}(0)-\tilde{\mathscr{E}}_{r}^{b}(0),
\end{aligned}
$$


where $\theta_{b}$ is the change of volume factor induced by the exponential map, i.e.,

$$
\theta_{b}(r)= \begin{cases}(\sin \sqrt{b} r / \sqrt{b} r)^{m-1} & \text { if } b>0 \\ 1 & \text { if } b=0 \\ (\sinh \sqrt{-b} r / \sqrt{-b} r)^{m-1} & \text { if } b<0\end{cases}
$$

Changing the variable from $r$ to $s(r)=F_{b}(r)$ defined in $(2.4)$ we get $\tilde{\mathscr{E}}_{R}^{b}(r)=$ $\tilde{\mathscr{E}}_{R}^{b}(r(s))=\mathbf{E}_{R}^{b}(s)$.

We shall need the following observations.

Proposition 4. $\mathbf{E}_{R}^{b}(s)$ is a smooth function of $s$ for $0 \leq s \leq s(R)$, and in this interval we have

$$
\begin{aligned}
& \frac{d}{d s} \mathbf{E}_{R}^{b}(s)<0 \quad \text { for every } b, \\
& \frac{d^{2}}{d s^{2}} \mathbf{E}_{R}^{b}(s) \begin{cases}<0 & \text { if } b>0, \\
=0 & \text { if } b=0, \\
>0 & \text { if } b<0 .\end{cases}
\end{aligned}
$$

Proof. Since $r(s)$ and $\tilde{\mathscr{E}}_{R}^{b}(r)$ (in (3.1)) are smooth when the respective arguments are nonzero, we see that $\mathbf{E}(s)$ is a smooth function of $s$ for $0<$ $s \leq s(R)$. The fact that $\tilde{E}_{R}^{b}(x)$ solves the Poisson equation $(1.1)$ on $\tilde{B}_{R}(\tilde{p})$ can therefore be written as

$$
-1=\tilde{\Delta} \tilde{E}=\mathbf{E}^{\prime \prime}(s) \cdot\left\|\operatorname{grad}_{\tilde{B}} s\right\|^{2}+\mathbf{E}^{\prime}(s) \cdot \tilde{\Delta} s,
$$

and so from (2.5),

$$
-1=\mathbf{E}^{\prime \prime}(s) \cdot\left(s^{\prime}(r)\right)^{2}+\mathbf{E}^{\prime}(s) \cdot m s^{\prime}(r) h_{b}(r),
$$

or equivalently

$$
-1=\mathbf{E}^{\prime \prime}(s) \cdot s(2-b s)+\mathbf{E}^{\prime}(s) \cdot m(1-b s) .
$$

The unique solution $\mathbf{E}(s)$ to (3.6) with $\mathbf{E}(0)=c_{0}$ has a power series expansion $\mathbf{E}(s)=\sum_{k=0}^{\infty} c_{k} s^{k}$ whose radius of absolute convergence is $2 /|b|$. Indeed, a substitution of the series into (3.6) leads to the recurrence relation

$$
c_{1}=-1 / m, \quad c_{k+1}=c_{k} \cdot b\left(k^{2}+(m-1) k\right) /\left(2 k^{2}+(m+2) k+m\right), \quad k \geq 1,
$$

and the claim now follows from the ratio test together with

$$
\left|c_{k+1}\right| /\left|c_{k}\right| \rightarrow|b| / 2 \quad \text { for } k \rightarrow \infty .
$$

In particular $\mathbf{E}_{R}^{b}(s)$ is therefore smooth in the closed interval $0 \leq s \leq s(R)$, and

$$
\begin{aligned}
\mathbf{E}_{R}^{b}(s) & -\mathbf{E}_{R}^{b}(0) \\
= & -\frac{1}{m} s-\frac{b}{2(m+2)} s^{2}-\frac{b^{2} \cdot(m+1)}{3(m+2)(m+4)} s^{3}-\cdots, \quad s<\frac{2}{|b|}
\end{aligned}
$$


We see that $\mathbf{E}^{\prime}(0)=-1 / m<0$, and the inequality (3.3) follows for all other values of $s$ from $r^{\prime}(s)>0$ and $\tilde{\mathscr{E}}^{\prime}(r)<0$ (from (3.1)). Furthermore, the series (3.7) also gives directly the statement (3.4) for $b>0$ and $b=0$ respectively. In case $b<0$ we first observe from $(3.7)$ that $\mathbf{E}^{\prime \prime}(0)=-b /(m+2)>0$. A differentiation of (3.6) with respect to $s$ gives

$$
0=\left(\mathbf{E}^{\prime \prime}(s)\right)^{\prime} \cdot s(2-b s)+\left(\mathbf{E}^{\prime \prime}(s)\right)(m+2)(1-b s)-m b \mathbf{E}^{\prime}(s) .
$$

Here $m b \mathbf{E}^{\prime}(s)$ is positive so that $\mathbf{E}^{\prime \prime}(s)=\eta(s)$ satisfies

$$
0<\eta^{\prime}(s) \cdot s(2-b s)+\eta(s) \cdot(m+2)(1-b s) .
$$

If a first zero $s_{0}$ exists for $\eta$, then $\eta^{\prime}\left(s_{0}\right)>0$, but this is clearly ruled out by $\eta(0)>0$. Hence $E^{\prime \prime}(s)$ is positive for all $s$ when $b<0$.

\section{Proof of Theorems 1 and 2}

The comparison of $E_{R}(x)$ with $\mathbf{E}_{R}^{b}\left(s\left(r_{p}(x)\right)\right)$ will follow from the identity

$$
\Delta_{\Omega} \mathbf{E}_{R}^{b}(s(r(x)))=\mathbf{E}^{\prime \prime}(s) \cdot\left\|\operatorname{grad}_{\Omega} s\right\|^{2}+\mathbf{E}^{\prime}(s) \cdot \Delta_{\Omega} s .
$$

In fact, suppose that $K_{N} \geq b>0$. Then by Corollary 3 (with $H_{\Omega}=0$ ), Proposition 4 and the fact that $\left\|\operatorname{grad}_{\Omega} s\right\|^{2} \leq\left\|\operatorname{grad}_{N} s\right\|^{2}=\left\|\operatorname{grad}_{\tilde{B}_{R}} s\right\|^{2}$ we get

$$
\begin{aligned}
\Delta_{\Omega} \tilde{E}_{R}^{b}(x) & \geq \mathbf{E}^{\prime \prime}(s) \cdot\left\|\operatorname{grad}_{N} s\right\|^{2}+\mathbf{E}^{\prime}(s) \cdot m s^{\prime}(r) h_{b}(r) \\
& \geq \mathbf{E}^{\prime \prime}(s) \cdot\left\|\operatorname{grad}_{\tilde{B}_{R}} s\right\|^{2}+\mathbf{E}^{\prime}(s) \cdot \tilde{\Delta}_{\tilde{B}_{R}} s \\
& =\tilde{\Delta}_{\tilde{B}_{R}} \tilde{E}_{R}^{b}=-1=\Delta_{\Omega} E_{R}(x) .
\end{aligned}
$$

Therefore $\tilde{E}_{R}^{b}(x)-E_{R}(x)$ is a subharmonic function on $\Omega_{R}$ vanishing on $\partial \Omega_{R}$ so that the maximum principle applies and gives $\tilde{E}_{R}^{b}(x) \leq E_{R}(x)$ for all $x \in \Omega_{R}$.

In case $K_{N} \leq b<0$ we get similarly

$$
\Delta_{\Omega} \tilde{E}_{R}^{b}(x) \leq \tilde{\Delta}_{\tilde{B}_{R}} \tilde{E}_{R}^{b}=-1=\Delta_{\Omega} E_{R}(x) .
$$

Thus $\tilde{E}_{R}^{b}(x)-E_{R}(x)$ is a superharmonic function vanishing on the boundary so that $\tilde{E}_{R}^{b}(x) \geq E_{R}(x)$ for all $x \in \Omega_{R}$. This proves Theorem 1 .

If $K_{N} \equiv b=0$, then by continuity $\tilde{E}_{R}^{b}(x)=E_{R}(x)$ for all $x \in \Omega_{R}$. Conversely, if $\tilde{E}_{R}^{b}(x)=E_{R}(x)$ for all $x \in \Omega_{R}$, and we are in one of the two cases of Theorem 1 with $b \neq 0$, then we conclude from $\left(\mathbf{E}_{R}^{b}(s)\right)^{\prime \prime} \neq 0$ that $\left\|\operatorname{grad}_{\Omega} s\right\|=\left\|\operatorname{grad}_{N} s\right\|$. Hence $\Omega_{R}$ is a minimal cone in $N$. If $N$ has constant curvature $K_{N} \equiv b \neq 0$, then by analytic continuation from $\Omega_{R}=\tilde{B}_{R}$ we finally get that all of $M^{m}$ is a totally geodesic submanifold of $N^{n}$. q.e.d. 
We note here that if $\Omega_{R}(p)$ is a codimension-0 domain of $N$ we get $\left\|\operatorname{grad}_{\Omega} s\right\|$ $=\left\|\operatorname{grad}_{N} s\right\|$ for free, so that the assumption of $E_{R}(x)=\tilde{E}_{R}^{b}(x)$ for all $x \in \Omega_{R}(p)$ now gives (from (4.1))

$$
\Delta_{\Omega} s=\tilde{\Delta}_{\tilde{B}_{R}} s \quad \text { for all } s .
$$

Under any of the curvature assumptions $\operatorname{Ric}_{N} \geq(n-1) b$ or $K_{N} \leq b,(4.2)$ is only possible if $K_{N} \equiv b$ along every 'radial' plane in $\Omega_{R}(p)$, which therefore must be isometric to the space form ball $\tilde{B}_{R}$ of constant curvature $b$ (cf. [1]). Thus we get the following rigidity theorem which completes the Corollaire in $[3$, p. 796].

Proposition 5. Let $\Omega_{R}^{n}(p)$ be a regular domain of $N^{n}$. Suppose that either $\operatorname{Ric}_{N} \geq(n-1) b$ or $K_{N} \leq b$ for some $b \in \mathbf{R}$, and that $E_{R}(x)=\tilde{E}_{R}^{b}(x)$ for all $x \in \Omega_{R}$. Then $\Omega_{R}$ is isometric to $\tilde{B}_{R}$ with constant curvature $b$.

\section{Submanifolds of $\mathbf{R}^{n}$}

Now suppose again that $\Omega_{R}$ is a regular domain of any submanifold of $\mathbf{R}^{n}$. Then

$$
\begin{aligned}
\Delta_{\Omega} \tilde{E}_{R}^{0}(x) & =\mathbf{E}^{\prime}(s) \cdot \Delta_{\Omega} s \\
& =\mathbf{E}^{\prime}(s) \cdot m s^{\prime}(r)\left(h_{0}(r)+p H_{\Omega}(x)\right) \\
& =\tilde{\Delta}_{\tilde{B}_{R}} \tilde{E}_{R}^{0}(x)+\mathbf{E}^{\prime}(s) \cdot m r(x) p H_{\Omega}(x) \\
& =\Delta_{\Omega} E_{R}(x)+m r(x) \mathbf{E}^{\prime}(s) \cdot p H_{\Omega}(x) .
\end{aligned}
$$

If we assume that $E_{R}(x)=\tilde{E}_{R}^{0}(x)$ for all $x \in \Omega_{R}$, then we must have $p H_{\Omega}(x) \equiv$ 0 .

To establish Theorem 3 stated in $\S 1$ we therefore only have to prove the following

Lemma 6. Let $M^{n-1}$ be an immersed hypersurface of $\mathbf{R}^{n}$. If $M$ has everywhere vanishing $p H$, then $M$ is minimal.

Proof. Suppose for contradiction that $H(q) \neq 0$ for some $q \in M$. Then $H \neq 0$ in some maximal neighborhood $\mathscr{U}(q) \subset M$. Since

$$
p H(x)=\left\langle\operatorname{grad}_{R^{n}} r, H\right\rangle=0,
$$

and $H$ is orthogonal to the tangent space $T_{x} \mathscr{U}$, we get $\operatorname{grad}_{R^{n}}(x) \in T_{x} \mathscr{U}$. Thus $Z(x)=\operatorname{grad}_{R^{n}} r(x)$ is a unit vector field whose straight line integral curves foliate $\mathscr{U}$. Hence $\mathscr{U}$ is part of a cone with vertex $p$. But on a cone in $\mathbf{R}^{n}$ the length of the mean curvature vector grows to $\infty$ as one approaches the vertex. In particular $\mathscr{U}(q)$ must contain $p$ where $\|H\|=\infty$. This contradicts the smoothness of $M$ and proves the lemma. 
STEEN MARKVORSEN

\section{References}

[1] J. Cheeger \& D. G. Ebin, Comparison theorems in Riemannian geometry, North-Holland Mathematical Library, Vol. 9, North-Holland, Amsterdam, 1975.

[2] S.-Y. Cheng, P. Li \& S.-T. Yau, Heat equations on minimal submanifolds and their applications, Amer. J. Math. 106 (1984) 1033-1065.

[3] A. Debiard, B. Gaveau \& E. Mazet, Ternps de sortie des boules normales et minoration locale de $\lambda_{1}$, C. R. Acad. Sci. Paris. Sér. A 278 (1974) 795-798.

[4] E. B. Dynkin, Markov processes, Vols. 1 and 2, Grundlehren Math. Wiss. Vols. 121 and 122, Springer, Berlin, (1965).

[5] A. Gray \& M. A. Pinsky, The mean exit time from a small geodesic ball in a Riemannian manifold, Bull. Sci. Math. (2) 107 (1983) 345-370.

[6] K. Jorge \& D. Koutroufiotis, An estimate for the curvature of bounded submanifolds, Amer. J. Math. 103 (1981) 711-725.

[7] L. Karp \& M. Pinsky, Mean exit time from an extrinsic ball of a hypersurface (Proc. Warwick Sympos. 1985, K. Elworthy, ed.), Pitman Research Notes in Math., No. 150, London, 1986, 179-186.

[8] S. Markvorsen, On the heat kernel comparison theorems for minimal submanifolds, Proc. Amer. Math. Soc. 97 (1986) 479-482.

The TeChNical University OF DenMaRK 